\title{
Radiofrequency facet joint denervation efficiency based on the severity of spondylarthrosis and in osteoporotic vertebral compression fractures. A retrospective study ${ }^{\star}$
}

\author{
Márton Balázsfi ${ }^{1, *}$, Dávid Kis, Tamás Tóth, Tamás Zsoldos, Pál Barzó \\ Department of Neurosurgery, Faculty of Medicine, University of Szeged, Csongrad County, Hungary
}

\section{A R T I C L E I N F O}

\section{Keywords:}

Radiofrequency denervation

Facet joint

Spondylarthrosis

Vertebral compression fracture

\begin{abstract}
A B S T R A C T
Objectives: Radiofrequency denervation of the facet joints is performed via a well-established method. Its primary, direct indication is a positive response to a nerve block injection (MBB). Our study aimed to find other, effective but indirect indication signs through the retrospective analysis of our patients treated earlier.

Patients and methods: In our institute between 1 January, 2008 and 31 December, 2017 facet joint denervation has been performed in more than 2000 cases, and we included 529 patients in our retrospective study. We had separate groups for vertebral compression fractures and for spondylarthrosis of different severity (Grade 1; 2-3; 4), thus we assessed the postoperative condition of these patients using Visual Analoge Scale (VAS). The efficacy of the intervention was examined in every groups separately according to symptoms and previous spine surgeries.

Results: In view of our results, chronic lumbago and dorsalgia that are attributable to osteoporotic vertebral compression fracture are obvious indications if they do not respond to conservative therapy, as $76.8 \%$ of such patients remained asymptomatic for minimum 6 months $(\mathrm{p}=0,000)$. Another indication is Grade 2 or 3 chronic spondylarthrosis without radicular involvement, since these groups reported a $51.4 \%$ success rate (asymptomatic for minimum 6 months) ( $p=0,015)$. Long term pain relief is obviously impaired by the presence of radicular compression, as we were not able to decrease the pain of $97 \%$ of such patients. Our findings also suggest that the vast majority of those who have previously undergone spine surgery cannot benefit from the intervention.

Conclusion: Based on this study, facet joint denervation can serve as an effective therapy supplement in a properly selected group of patients who do not respond to oral NSAIDs, exercise and physiotherapy. By this procedure we found we can reach long term benefit in the groups of osteoporotic vertebral fracture patients and patients with moderate spondylarthrosis. According to our results and the literature datas the properly patient selection for the indication of the RF ablation can be as effective as the controversial diagnostic nerve block injections.
\end{abstract}

\section{Introduction}

The prevalence of lower back pain generated by facet joint disorders is based on the efficiency of diagnostic nerve block injections. In the USA, long-term improvement could be achieved in $7.7-75 \%$ of patients with lower back pain, which confirms facet joint involvement [1,2]. According to another study, $15 \%$ of American workers treated for chronic lower back pain gave a positive response to nerve block injections [3]. A similar study states that the prevalence of pain generated by the facet joints is $40-45 \%$ in pain management $[4,5]$ and $40 \%$ in the Australian rheumatologic practice [6].

The efficacy of nerve block injections is controversial worldwide. As the case count at our facility exceeded 2000 and the procedure is almonthst invariably free of complications, our intervention included the

\footnotetext{
${ }^{\star}$ Portions of this work were presented at the Congress of the Hungarian Pain Society, Szeged, Hungary, November 12, 2016 and Congress of Hungarian Neurosurgical Society, Miskolc, Hungary, November 18, 2016.

* Corresponding author.

E-mail address: marton.balazsfi@gmail.com (M. Balázsfi).

${ }^{1}$ Postal Address of the Corresponding Author: Department of Neurosurgery, Faculty of Medicine, University of Szeged, ${ }^{6 \text { th }}$ Semmelweis Street, Szeged, H-6725, Hungary.
} 
physical examination of patients and the analysis of their MRI scans. Since the majority of the scans were not available at the time of the follow-up analysis, our retrospective study included only 519 of the more than 2000 cases.

\section{Patients and methods}

\subsection{Patient selection}

We performed therapeutic radiofrequency (RF) denervation of thoracic or lumbar facet joints in the following cases:

- The symptoms and signs suggested thoracic or lumbar axial, paravertebral or radicular pain arising from a facet joint;

- The involvement of the facet joint was confirmed with imaging scans

- Conservative therapy (including NSAIDs, physiotherapy and home exercises) of over 3 months did not relieve the pain;

- The patient did not undergo similar facet joint RF denervation in the previous 3 months [25].

Exclusion criteria: if the pain was caused by neoplasia (differential diagnosis: MRI DWI) [26]; if the patient had an intracardiac pacemaker implanted; if the patient had coagulopathy or took anticoagulants; could not stay still in the prone position for at least $30 \mathrm{~min}$.; or local infection.

\subsection{Technical background}

At our facility, we perform continuous RF ablation in the following way: after placement of the electrode the clinician initially stimulates the pain fibres at $50 \mathrm{~Hz}$ at a pulse width of $1 \mathrm{~ms}$. As the voltage is increased to a maximum of $2 \mathrm{~V}$, the patient experiences pain and tension at the stimulated site and reports a pseudoradicular referral pattern. In the next step, the motor fibres are stimulated at $2 \mathrm{~Hz}$ at a pulse width of $1 \mathrm{~ms}$, while the voltage is increased to a maximum of $3 \mathrm{~V}$. The test is positive if muscle jerks develop in the lower extremity, too. In that case, the electrode is situated too close to the motor fibres, thus its repositioning is necessary in order to avoid unwanted damage to these fibres during the ablation. As long as the above mentioned muscle jerks are not present, denervation is performed through a one-minute ablation at $80^{\circ} \mathrm{C}$. Finally, $40 \mathrm{mg}$ methylprednisolone (Depo-Medrol ${ }^{\circledR}$ ) is injected to the site of the intervention. Prior to discharge, the patients are observed for another $1-2 \mathrm{~h}$ during which time they receive no analgesia.

\subsection{Assessment}

We performed a retrospective analysis of the MRI scans. The degree of facet joint degeneration was graded according to the severity on imaging. Grade 1 - normal joint; grade 2 - narrowing of the joint space, as well as small osteophytes; grade 3 - sclerosis or moderate

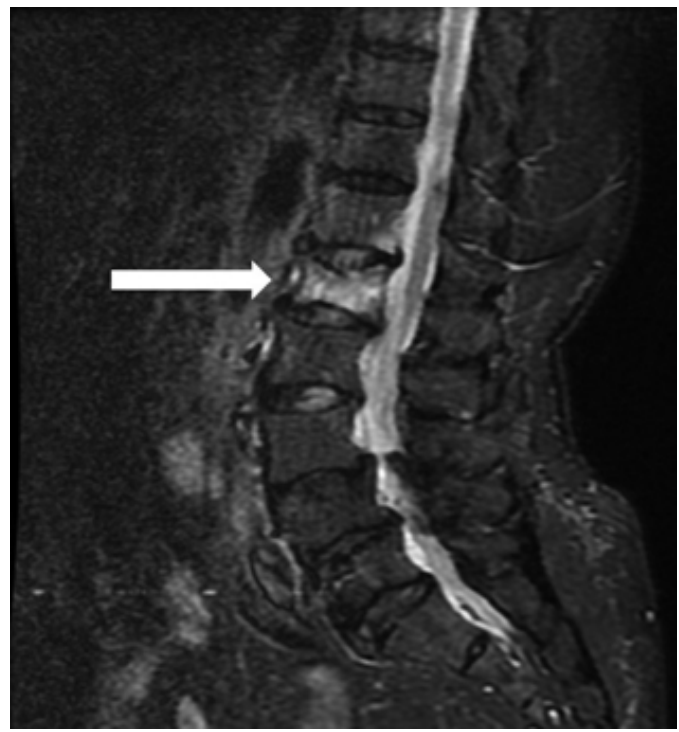

Fig. 2. Thoracolumbar spine MRI STIR examination. The arrow shows the compression fracture of the L.I. vertebra with oedema.

osteophytes; grade 4 - large osteophytes [16]. Marked osteophyte formation can even narrow the intervertebral foramen [12] (Fig. 1). If the underlying condition was compression fracture of the vertebra, it was detected with two-dimensional x-ray or CT/ MRI scans if necessary (Fig. 2).

Our retrospective study included a total of 529 patients (384 females, 145 males). The youngest patient was 26 , whereas the oldest was 89 years old. The mean age of participants was 57 years. During the preoperative examination, history taking was followed by physical examination, and in some cases the examination suggested involvement of the facet joints. The MRI scan we performed in all cases confirmed the involvement of the facet joints, which was graded accordingly (grades 1-4). Grade 1: no spondylarthrosis (38 cases); Grades 2-3: spondylarthrosis without radicular involvement (206 cases); Grade 4: spondylarthrosis with radicular involvement (177 cases). Patients with osteoporotic vertebral fractures were assigned to a separate group (108 cases). The severity of the pain was assessed with the Visual Analogue Scale before the intervention and postoperatively at 2, 6 and 12 months. Changes in referred lower back and pseudoradicular pain were assessed separately. We evaluated the changes of the radicular pain in patients with grade 4 spondylarthrosis. Patients with osteoporotic vertebral fractures were further divided into 2 groups: (a) previous vertebroplasty (30 cases) and (b) no previous surgery (78 cases). The spondylarthrosis groups (grade 1-4) were also divided into subgroups based on their surgical history (whether they had a previous spine surgery or not). The type of the operation (laminotomy;
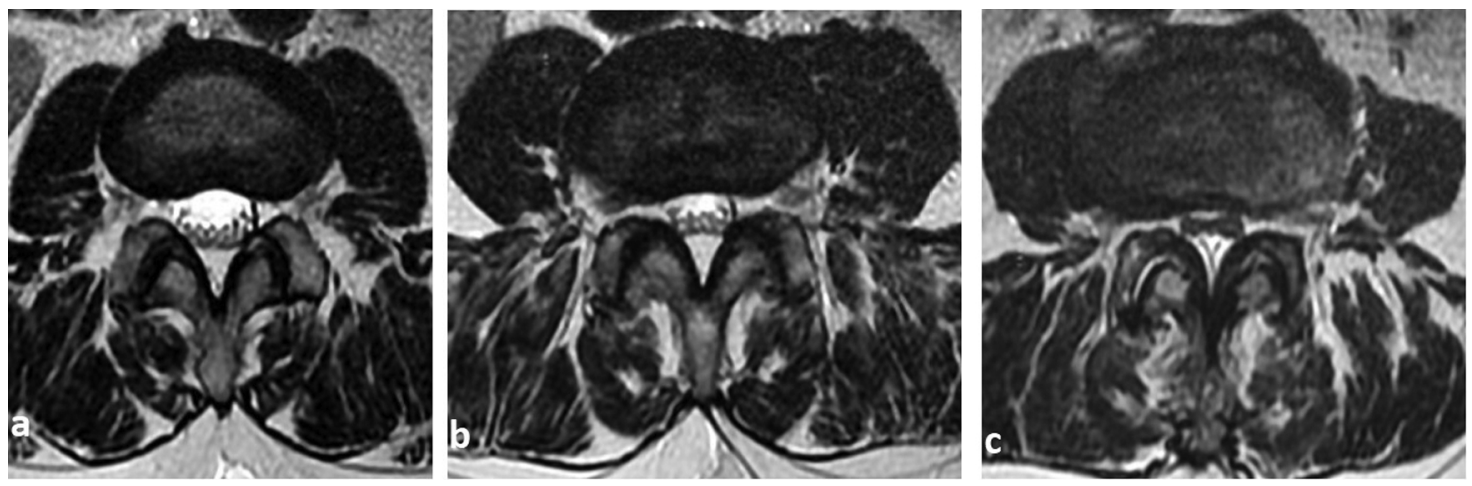

Fig. 1. Grades of the spondylarthrosis - a: grade 1; b: grade 2-3; c: grade 4 . 
hemilaminectomy-laminectomy; stabilisation and interspinosus implantation) was also taken into consideration.

In Grade 1 patients, whose imaging scan did not reveal spondylarthrosis but whose symptoms suggested facet joint involvement and exercises or physiotherapy did not improve the symptoms, the intervention was performed as a means of potential pain relief. In Grade 4 patients with spodylarthrosis, facet joint denervation was considered if besides the referred pain their mainly complained about lower back pain attributable to facet joint degeneration or if open surgical decompression was not feasible due to the general condition of the patient or to the obvious lack of symptoms.

Before the intervention, the pain sensation of patients was assessed with the Visual Analogue Scale (VAS). At two months, we used the same instrument to estimate the severity of the pain, and we asked detailed questions concerning changes in pain severity, the amount of NSAIDs taken and fitness to work. The patients were evaluated again at 6 and 12 months.

The intervention was considered successful if the VAS showed a 50percent decrease in pain sensation, or if the patient consumed significantly fewer NSAIDs or if they could switch to a weaker class of active agents.

We used Chi-Square test and SPSS program for statistical analysis.

\section{Results}

The intervention proved to be the months successful in relieving the pain caused by osteoporotic vertebral fractures (108 cases). In $76.8 \%$ of the cases (83 out of 108 cases) we could achieve a significant pain reduction ( $\mathrm{p}=0,000$ ) for at least 6 months, and in 39 of the 83 patients pain relief was effective for minimum one year, which accounts for $36.1 \%$ of the cases. We noted a significant difference between the outcome of those who were not operated on after the fracture and those who underwent vertebroplasty. In the conservative therapy group, $63.8 \%$ (69 cases) of patients experienced improvement of at least 6 months, and $25.9 \%$ (28 cases) remained asymptomatic for a year. However, only $12.9 \%$ (14 cases) of the surgical group experienced the same improvement. There was no prominent differences between the efficacy of the ablation of the LBP and the Pseu group (Table 1 and Fig. 3).

Similar positive results were achieved in grade 2 and 3 spondylarthrosis group (206 cases) (Table 2 and Fig. 4). In $51.4 \%$ of the cases (106 cases), we could achieve significant pain reduction $(\mathrm{p}=0,015)$ for at least 6 months, and in 56 patients pain relief was effective for minimum one year, which accounts for $27.1 \%$ of the cases. In patients who had had no previous surgery, appropriate pain relief of minimum 6 months was achieved in $69.9 \%$ of the cases (93 of 133 cases) $(\mathrm{p}=0,000)$. In the case of facet joint pain following laminotomy (26 cases) $38.5 \%$ of patients (10 cases) reported improvement of at least 6 months, and $80 \%$ of them ( 8 cases) remained asymptomatic for a year. However, in the group where the previous spinal surgery had involved facet joint resection (hemilaminectomy, laminectomy, stabilisation) (39 cases) $91.6 \%$ of the interventions proved to be ineffective; appropriate
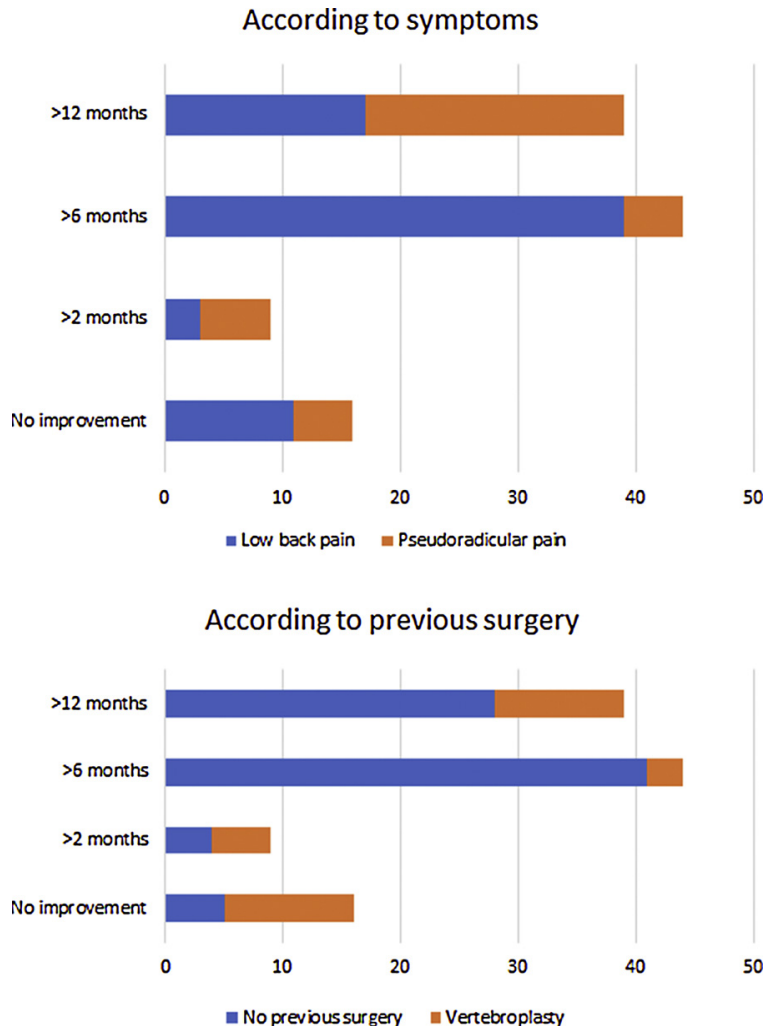

Fig. 3. The diagram shows long-term analgetic effect of facet joint denervation in osteoporotic vertebral fracture (108 cases) All of the abbrevations are removed.

pain relief was achieved for 2 months in 3 patients and another 3 subjects remained asymptomatic for 6 months. Patients with interspinosus implants experienced a two-month improvement in $37.5 \%$ of the cases ( 3 of 8 cases) before the pain returned.

When confirmed spondylarthrosis resulted in stenosis of the radicular canal or the lateral recess and subsequent radicular compression, the symptoms were not invariably of radicular origin (177 cases) (Table 3). Five patients experienced only low back pain and lower back pain was associated with pseudoradicular pain in another 5 cases; however, we could not achieve long-term improvement in any of these patients. Out of 167 subjects with lumbar ischialgia (Low back pain and radicular pain), only 32 reported satisfactory pain relief, which accounts for a $19.2 \%$ success rate. At the two-month follow-up $8.1 \%$ (11) of patients reported improvement in their lower back pain, and $9.6 \%$ (16 cases) stated that their referred pain had been alleviated ( $\mathrm{p}=0,000$ ). Long-term pain relief of minimum 6 months was successful in only 5 patients $(3 \%)$. As regards classification based on previous surgery, this group did not show any differences. The intervention was ineffective in $96,7 \%$ ( 88 cases) of cases where the patient had no previous open spine surgery (91 cases) but the MRI scan confirmed

Table 1

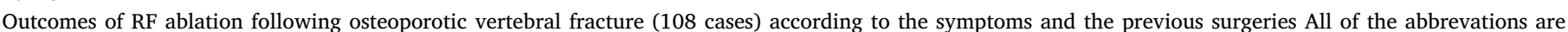
removed.

\begin{tabular}{|c|c|c|c|c|c|}
\hline \multicolumn{6}{|l|}{ Symptoms } \\
\hline & & No improvement & $>2$ months & $>6$ months & $>12$ months \\
\hline & Low back pain & 11 & 3 & 39 & 17 \\
\hline & Pseudoradicular pain & 5 & 6 & 5 & 22 \\
\hline \multicolumn{6}{|c|}{ Previous surgery } \\
\hline & & No improvement & $>2$ months & $>6$ months & $>12$ months \\
\hline & No previous surgery & 5 & 4 & 41 & 28 \\
\hline & Vertebroplasy & 11 & 5 & 3 & 11 \\
\hline
\end{tabular}


Table 2

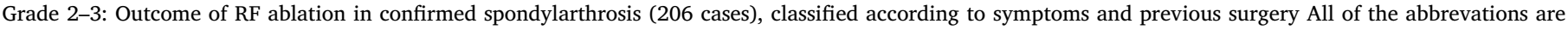
removed.

\begin{tabular}{|c|c|c|c|c|c|}
\hline \multicolumn{6}{|l|}{ Symptoms } \\
\hline & & No improvement & $>2$ months & $>6$ months & $>12$ months \\
\hline & Low back pain & 39 & 9 & 22 & 11 \\
\hline & Pseudoradicular pain & 45 & 7 & 28 & 45 \\
\hline \multicolumn{6}{|c|}{ Previous surgery } \\
\hline & & No improvement & $>2$ months & $>6$ months & $>12$ months \\
\hline & No previous surgery & 35 & 5 & 45 & 48 \\
\hline & Laminotomy & 11 & 5 & 2 & 8 \\
\hline & Laminectomy & 17 & 1 & 2 & - \\
\hline & Stabilisation & 16 & 2 & 1 & - \\
\hline & Interspinosus implantation & 5 & 3 & - & - \\
\hline
\end{tabular}

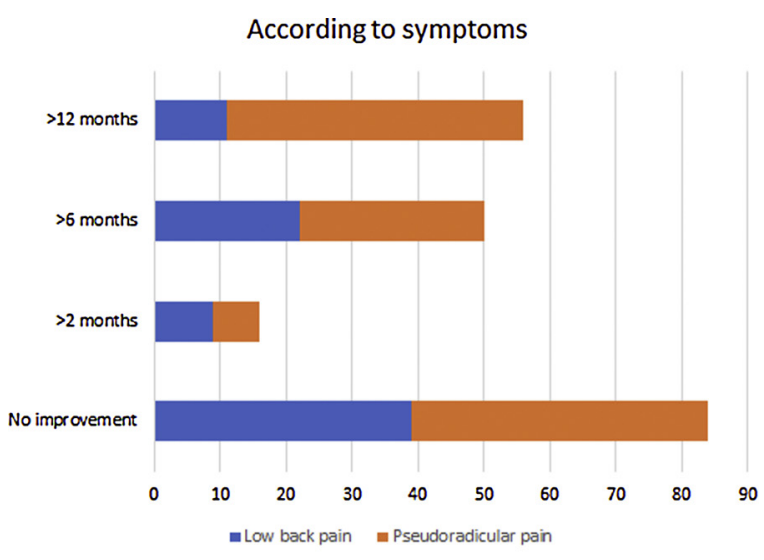

According to previous surgery

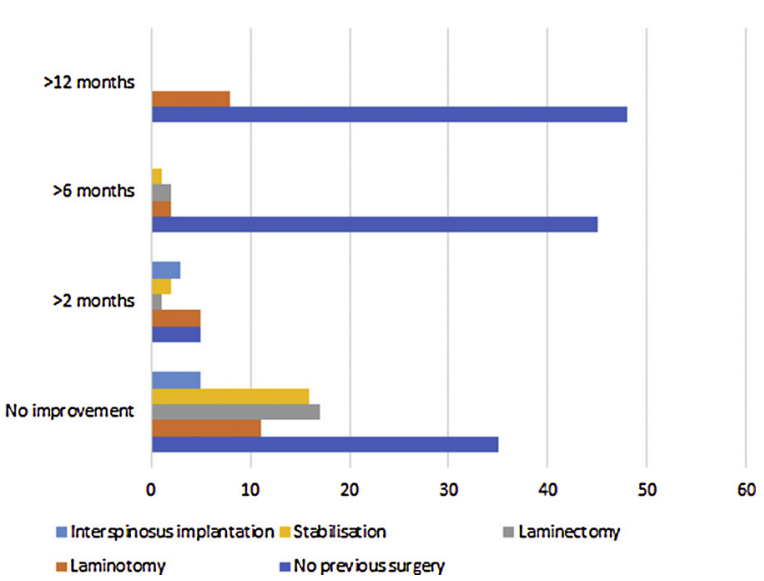

Fig. 4. The diagram shows long-term analgetic effect of facet joint denervation in Grade 2-3 spondylarthrosis (206 cases), classified according to symptoms and previous surgery All of the abbrevations are removed.

radicular compression. Two-month pain relief was achieved in $24 \%$ of the cases (22 cases), and only 3 patients $(3,2 \%)$ were asymptomatic at the six-month follow up. In this group, hardly any patients with a previous open spine surgery (86 cases) could achieve effective, longterm pain relief. Only 2 patients reported positive outcomes at 6 months, which means that pain relief was ineffective in $98 \%$ of the cases.

In Grade 1 spondylarthrosis (38 cases) (Table 4), where the physical examination suggested facet joint involvement but it was not confirmed by imaging scans, only $15.8 \%$ of patients ( 6 cases) could achieve longterm pain relief $(p=0,021)$. Seventy-one percent of the patients experienced no improvement at all. None of the 6 patients who reported a minimum of 6 months' improvement had had a previous spine surgery.

\section{Discussion}

\subsection{Diagnostic assessment of nerve block injections}

Patients who responded to diagnostic nerve block injections (MBB $=$ medial branch block) experienced less intensive pain on hyperextension and on extension-rotation [7]. Some studies could not find any connection between patients responding to MBB injections and patients who develop pain in an upright, standing position or whose pain sensation can be provoked with lumbar extension [8].

Another clinical sign that suggests lower back pain of facet joint origin is the lack of referred pain that spreads beside the knees. Such pain suggests radicular irritation and the generated pain corresponds to its dermatome in the lower limb, while pain originating from the facet joints corresponds to the sclerotome of the affected level. This is also confirmed by a test showing that a significantly higher proportion of patients who did not respond to MBB complained of referred lower extremity pain than those who did respond [9]. However, two bigger studies could not confirm this finding, as they did not find any relationship between the 2 symptoms $[7,10]$. In addition, in patients with pain of facet joint origin who also complained of a large area of referred pain osteoarthritis of the facet joints was detected in several cases [11].

When a group of patients who responded to intraarticular nerve block injections were subsequently given intraarticular steroid injections, they did not experience a more significant improvement than the placebo group [13]. The contrary was found in patients who underwent diagnostic MBB injections followed by RF denervation. The retrospective study of Steven P. Cohen et al. (2008) analysed 262 patients from 3 pain centres and they found no relationship between the level of pain relief following MBB injections and long-term (6 months) outcomes following RF ablation $[14,15]$.

\subsection{The correlation between vertebral compression fractures and pain of facet joint origin}

The number of osteoporotic vertebral fractures increases with age, especially among women as its prevalence over 50 years of age is $26 \%$, whereas over 80 it reaches $40 \%$ [17]. Compression fractures cause pain, and in severe cases they are associated with neurological symptoms. In a high number of cases, transpendicular screw fixation is not feasible due to the weak and delicate bone mass, not to mention the effects of prolonged general anaesthesia on comorbitities and the potential complications associated with the prone position during surgery [18]. Percutaneous vertebroplasty and kyphoplasty are effective at vertebral body reconstruction, pain reduction and stabilisation of the affected 
Table 3

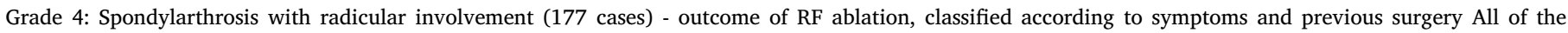
abbrevations are removed.

\begin{tabular}{|c|c|c|c|c|c|}
\hline \multicolumn{6}{|l|}{ Symptoms } \\
\hline & & No impovement & $>2$ months & $>6$ months & $>12$ months \\
\hline & Low back pain & - & 5 & - & - \\
\hline & Pseudoradicular pain & 5 & - & - & - \\
\hline & Low back and radicular pain & 135 & $11+16$ & $1+4$ & - \\
\hline \multicolumn{6}{|c|}{ Previous surgery } \\
\hline & & No impovement & $>2$ months & $>6$ months & $>12$ months \\
\hline & No previous surgery & 66 & 22 & 3 & - \\
\hline & Laminotomy & 39 & 7 & 2 & - \\
\hline & Laminectomy & 11 & 2 & - & - \\
\hline & Stabilisation & 22 & - & - & - \\
\hline & Interspinosus implantation & 2 & 1 & - & - \\
\hline
\end{tabular}

segment of the spine, and the duration of the intervention is significantly shorter, which allows for a short narcosis or even local anaesthesia, thereby decreasing the risk of severe complications [19].

The collapse of the vertebral body decreases the anterior height of the spine, therefore the posterior supporting elements, namely the facet joints will need to cope with more loading, which can result in persistent axial or pseudoradicular pain postoperatively [20].

Percutaneous vertebroplasty and kyphoplasty are effective at reducing acute pain; however, chronic pain that persists in spite of physiotherapy tends to originate from the facet joints [21]. Facet joint blocks can be beneficial in such cases as they can achieve significant pain reduction and can enable patients to continue physiotherapy sessions [22].

In the elderly, chronic dorsalgia or lumbago are often attributable to a prior vertebral compression fracture which is only revealed during the investigation. This type of pain originates from the muscles, ligaments or facet joints rather than from the vertebral body [23]. Intraarticular steroid injections can also effectively reduce pain persisting after vertebro- and kyphoplasty [20].

The question is whether a significant proportion of acute pain may be attributed to facet joint origin. A study that compared vertebroplasty, kyphoplasty and medicinal facet joint denervation in the treatment of vertebral compression fractures found that cement augmentation is significantly more effective at acute (one week) pain reduction; however, the long-term outcome of the procedures did not turn out to be different [24] Consequently, medicinal analgesia and immobilisation seem to be effective for the treatment of acute pain, while chronic dorsalgia and lumbago probably respond to intraarticular blocks, which may be RF denervation of the medial branch.

\section{Conclusions}

In view of our findings and literature data, we can state that lower back pain of osteoporotic or traumatic origin is an appropriate indication for facet joint denervation if the pain is due to vertebral compression that does not necessitate surgical intervention. In our study, $76.8 \%$ of the patients reported significant pain reduction for min. 6 months. RF ablation is less effective at relieving residual pain following vertebro- or kyphoplasty. In Grade 2-3 spondylarthrosis, we found significant, long-term pain relief in $51.4 \%$ of our patients.

Pain relief is obviously impaired by the presence of radicular compression, as we were not able to decrease the pain of $97 \%$ of such patients. Our findings also suggest that the vast majority of those who have previously undergone spinal surgery cannot benefit from the intervention. Our results were not obviously positive either when the imaging studies did not confirm facet joint involvement. In patients with a prior stabilisation surgery, the intervention was only successful in the adjacent segment syndrome, as in this case the segment above the stabilised region was affected by facet joint degeneration due to overload. Our results also suggest that a second intervention in patients who found the first denervation effective but later experienced partial relapse can be associated with a pain reduction of several years.

According to our results and the literature datas the properly patient selection for the RF ablation can be as effective as the controversial diagnostic nerve block injections.

\section{Appendix A. Supplementary data}

Supplementary material related to this article can be found, in the online version, at doi:https://doi.org/10.1016/j.clineuro.2019.105497.

Table 4

Grade 1: MRI did not confirm spondylarthrosis but the symptoms suggest facet joint involvement (38 cases) - outcomes of RF ablation classified according to symptoms and previous surgery All of the abbrevations are removed.

\begin{tabular}{|c|c|c|c|c|c|}
\hline \multicolumn{6}{|l|}{ Symptoms } \\
\hline & & No improvement & $>2$ months & $>6$ months & $>12$ months \\
\hline & Low back pain & 5 & 2 & - & 5 \\
\hline & Pseudoradicular pain & 10 & 1 & - & - \\
\hline & Radicular pain & 12 & 2 & 1 & - \\
\hline \multicolumn{6}{|c|}{ Previous surgery } \\
\hline & & No improvement & $>2$ months & $>6$ months & $>12$ months \\
\hline & No previous surgery & 5 & 2 & 1 & 5 \\
\hline & Laminotomy & 12 & 1 & - & - \\
\hline & Laminectomy & 2 & - & - & - \\
\hline & Stabilisation & 6 & - & - & - \\
\hline & Interspinosus implantation & 2 & 2 & - & - \\
\hline
\end{tabular}




\section{References}

[1] J. Dreyer, P.H. Dreyfuss, Low back pain and the zygapophysial (facet) joints, Arch. Phys. Med. Rehabil. 77 (3) (1996) 290-300.

[2] L. Kalichman, L. Li, D.H. Kim, et al., Facet joint osteoarthritis and low back pain in the community-based population, Spine Phila Pa 197633 (23) (2008) 2560-2565.

[3] A.L. Nachemson, Newest knowledge of low back pain: a critical look, Clin. Orthop. Relat. Res. (279) (1992) 8-20.

[4] L. Manchikanti, V. Pampati, B. Fellows, C.E. Bakhit, Prevalence of lumbar facet joint pain in chronic low back pain, Pain Phys. 2 (3) (1999) 59-64.

[5] L. Manchikanti, V. Pampati, B. Fellows, C.E. Bakhit, The diagnostic validity and therapeutic value of lumbar facet joint nerve blocks with or without adjuvant agents, Curr. Rev. Pain 4 (5) (2000) 337-344.

[6] A.C. Schwarzer, S.C. Wang, N. Bogduk, P.J. McNaught, R. Laurent, Prevalence and clinical features of lumbar zygapophysial joint pain: a study in an Australian population with chronic low back pain, Ann. Rheum. Dis. 54 (2) (1995) 100-106.

[7] M.E. Revel, V.M. Listrat, X.J. Chevalier, M. Dougados, M.P. N'guyen, C. Vallee, M. Wybier, F. Gires, B. Amor, Facet joint block for low back pain: identifying predictors of a good response, Arch. Phys. Med. Rehabil. 73 (1992) 824-828.

[8] A.C. Schwarzer, C.N. Aprill, R. Derby, J. Fortin, G. Kine, N. Bogduk, Clinical features of patients with pain stemming from the lumbar zygapophysial joints, Spine 19 (10) (1994) 1132-1137.

[9] J.C.T. Fairbank, W.M. Park, I.W. McCall, J.P. O'Brien, Apophyseal injection of local anesthetic as a diagnostic aid in primary low-back pain syndromes, Spine 6 (6) (1981) 598-605.

[10] J.C.T. Fairbank, W.M. Park, I.W. McCall, J.P. O'Brien, Apophyseal injection of local anesthetic as a diagnostic aid in primary low-back pain syndromes, Spine 6 (6) (1981) 598-605.

[11] A.C. Schwarzer, S.-C. Wang, D. O’Driscoll, T. Harrington, N. Bogduk, R. Laurent, The ability of computed tomography to identify a painful zygapophysal joint in patients with chronic low back pain, Spine 20 (8) (1993) 907-912.

[12] Gwen Sowa, Facet-mediated pain, Dis Mon. 51 (2005) 18-33.

[13] N. Bogduk, A narrative review of intra-articular corticosteroid injections for low back pain, Pain Med. 6 (2005) 287-296.

[14] Nikolai Bogduk, Evidence-informed management of chronic low back pain with facet injections and radiofrequency neurotomy, Spine J. 8 (2008) 56-64.
[15] Steven P. Cohen, Lumbar zygapophysial (facet) joint radiofrequency denervation success as a function of pain relief during diagnostic medial branch blocks: a multicenter analysis, Spine J. 8 (2008) 498-504.

[16] A. Fujiwara, K. Tamai, M. Yamato, The relationship between facet joint osteoarthritis and disc degeneration of the lumbar spine: an MRI study, Eur. Spine J. 8 (1999) 396-401.

[17] L.J. Melton 3rd, D.F. Kallmes, Epidemiology of vertebral fractures: implications for vertebral augmentation, Acad. Radiol. 13 (2006) 538-545.

[18] A. Rapado, General management of vertebral fractures, Bone 18 (3 Suppl) (1996) 191S-196S.

[19] N.B. Watts, S.T. Harris, H.K. Genant, Treatment of painful osteoporotic vertebral fractures with percutaneous vertebroplasty or kyphoplasty, Osteoporos. Int. 12 (2001) 429-437.

[20] R. Mitra, H. Do, T. Alamin, I. Cheng, Facet pain in thoracic compression fractures, Pain Med. 11 (2010) 1674-1677.

[21] S.R. Garfin, H.A. Yuan, M.A. Reiley, New technologies in spine: kyphoplasty and vertebroplasty for the treatment of painful osteoporotic compression fractures, Spine (Phila Pa 1976) 26 (2001) 1511-1515.

[22] K.D. Park, H. Jee, H.S. Nam, S.K. Cho, H.S. Kim, Y. Park, O.K. Lim, Effect of medial branch block in chronic facet joint pain for osteoporotic compression fracture: one year retrospective study, Ann. Rehabil. Med. 37 (April (2)) (2013) 191-201.

[23] B.A. Georgy, Interventional techniques in managing persistent pain after vertebral augmentation procedures: a retrospective evaluation, Pain Phys. 10 (2007) 673-676.

[24] Biao Wang, Hua Guo, Li Yuan, Dageng Huang, Haiping Zhang, Dingjun Hao, A prospective randomized controlled study comparing the pain relief in patients with osteoporotic vertebral compression fractures with the use of vertebroplasty or facet blocking, Eur. Spine J. (2016) Feb 5.

[25] Leena Niemistö, Eija Kalso, Antti Malmivaara, Seppo Seitsalo, Heikki Hurri, Radiofrequency denervation for neck and back pain: a systematic review within the framework of the cochrane collaboration back review group, Spine 28 (2003) 1877-1888 MD, PhD Number 16.

[26] A. Baur, A. Stabler, R. Bruning, Diffusion-weighted MR imaging of bone marrow: differentiation of benign versus pathologic compression fractures, Radiology 207 (1998) 349-356. 